\title{
The combined solution C04 for Earth Orientation Parameters consistent with International Terrestrial Reference Frame 2005
}

\author{
Christian Bizouard, Daniel Gambis \\ Observatoire de Paris, SYRTE, 61 av. de l'Observatoire, Paris, France \\ contact : christian.bizouard@obspm.fr
}

\section{Abstract}

The Earth Orientation Center of the IERS, located at Paris Observatory, SYRTE, has the task to provide to the scientific community the international reference time series for the Earth Orientation Parameters (EOP), referred as "IERS C04" (Combined 04), resulting from a combination of operational EOP series, each of them associated with a given geodetic technique. The procedure developed to derive the C04 solution was recently upgraded for several reasons: first we have implemented the new IAU2000 conventions; secondly it has been necessary to re-align the solution to improve its consistency with respect to the ITRF. Due to the separate determination of both celestial and terrestrial reference frames and EOP, there has been a slow degradation of the overall consistency and discrepancies at the level of 300 micoarseconds were existing between the current IERS C04 and the ITRF realization. We have taken this opportunity to upgrade the numerical combination procedure; improvements concern in particular routines, tables dimensions, generalized double precisions. Using the combined polar motion solution associated with the newly release International Terrestrial Reference Frame 2005 (ITRF 2005), we produce a better solution including estimates of the errors of combined values. Individual EOP series have been reprocessed since 1984. Pole coordinates are now fully consistent with ITRF. The nuta- tion offsets and UT1 are made consistent with the International Celestial Reference Frame (ICRF) through the IVS combined solution. The new $\mathrm{C} 04$ solution, referred as 05 C04, updated two times per week like the official solution, is provisional and is in validation process by IERS experts, until it becomes the OFFICIAL C04 solution.

\section{Introduction}

The Earth Orientation Parameters (EOP) describe the irregularities of the Earth rotation with respect to a non-rotating reference frame. Two parameters (dpsi,deps) correct the precession-nutation model of the celestial pole, one parameter $(U T 1-U T C)$ gives the irregularities of the rotation angle, and the two last one $(x, y)$ describe the polar motion with respect to the crust. They give the full transformation between the International Terrestrial Reference Frame (ITRF) and the International Celestial Reference Frame (ICRF). The reference EOP series computed at the Earth Orientation Center at Paris Observatory is obtained from the combination of "operational" EOP series derived from the various astro-geodetic techniques : Laser Ranging to the Moon (LLR) and to dedicated artificial satellites (SLR), Very Large Baseline Interferometry on extragalactic sources (VLBI) and more recently from GPS and DORIS systems (Gambis, 2004). The "combination" performed twice a week (Tuesdays and Thursdays) consists in 
series given at one-day intervals for each of those parameters.

The objective of this paper is to twofold : 1) present the $\mathrm{C} 04$ combination procedure and the recent improvements brought in the software code 2) present the new EOP C04 solution, its accuracy, and how it is made consistent with ITRF 2005.

\section{Description of the proce- dure}

Step 1 : Selection of a set of operational series, rescaling of the formal uncertainties and realigment in the ITRF system. After selecting the operational series to be combined, we rescale their formal uncertainties. Applying the "Threecornered Hat" method involving the whole set we determine the coefficients (one per series) by which the formal uncertainties of the series has to be multiplied in order to get more realistic estimates.

Step 2: EOP series are made consistent with the ICRF and ITRF. One of the main tasks of the combination is to produce EOP series consistent with the International Celestial Reference Frame (ICRF) and the International Terrestrial Reference Frame (ITRF). The operational series are not perfectly aligned with the ITRF and ICRF. They are often referred to different terrestrial and celestial systems, realized by the Analysis Centers. As it can be easily shown, the inconsistency of the EOP series with respect to the ITRF and ICRF produce systematic errors between series (Zhu and Mueller, 1983, IERS Annual Report 2000). In the late eighties, inconsistencies were as large as 1 mas, they are now reduced to 200 $\mu$ as for (x,y), $10 \mu \mathrm{s}$ for UT1, and $50 \mu$ as for (dpsi,deps) but they are still significant.

Before the combination is performed, it is necessary to translate all series into the ICRF and ITRF. We assume:
- that the celestial pole offsets (dPsi, dEps) provided by the IVS combined solution give the direction of the CIP in the ICRF without any significant drift ;

- that the UT1 values of the EOP (ITRF) 2005 combined solution gives the rotation angle of the ITRF with respect to ICRF without any significant drift from 1993 to 2007 ;

- that the polar motion (x,y) associated to the ITRF 2005 solution gives the direction of the CIP in the ITRF without any linear trend.

In order to ensure these translations, we assume that some "outstanding" series are already consistent with ITRF or ICRF. The drifts between "ITRF/ICRF consistent series" and operational series are not perfectly linear over several years, and we model them as broken lines, that are consecutive linear trends. For each operational series we estimate the linear drift (bias + trend) according to the schedule reported in Table 1. The estimated drifts are then removed from the operational EOP, which become "consistent" with the ITRF and ICRF, and are ready for combination.

Step 3: Differences : Operational series - intermediate reference. We do not directly combine the initial values of the series. The more these values vary, the larger will be the error introduced by interpolation, filtering, and any kind of numerical calculation. Therefore, we have to remove from the operational EOP series a well know reference, containing the main part of the signal. This reference is nothing else than the former combined solution obtained in a previous run, extended by a prediction. To achieve this, the reference series are interpolated for each date of the operational series using Lagrange interpolation over four points. The difference between operational series and reference series is then made. 


\begin{tabular}{ccc}
\hline EOP & Time interval & Reference Series \\
\hline dPsi / dEps & $1984-1993$ & IVS combined solution \\
& $1993-2007$ & id \\
\hline UT1 & $1984-1993$ & EOP ITRF 2005 (IGN) \\
\hline \multirow{3}{*}{ x,y } & $1993-2007$ & EOP ITRF 2005 (IGN) \\
& $1984-1993$ & IVS combined solution \\
& $1993-2000$ & EOP ITRF 2005 (IGN) \\
& $2000-2006$ & id
\end{tabular}

Table 1: Reference series according to the epoch of the solution

The analyses we have performed show that the differences between individual series and the reference is characterized by white noise. The combination process is applied on these differences.

Some characteristics:

- For the offsets of nutation, the parameters of the reference series are dPsi, dEps referred to IAU 2000 precessionnutation model. Therefore all "operational" celestial pole offsets are transformed into (dpsi,deps)/IAU 2000, before differencing.

- UT1-UTC present jumps (because of leap seconds), unconvenient for numerical treatment. Therefore we start to produce the time series UT1-TAI by taking into account all the leap seconds.

Step 4: Trend of LOD determined by satellite techniques is made consistent with UT1 observed by VLBI The trend of the LOD series derived from GPS and SLR series cannot be trusted: because of non-modeled instabilities in the satellite orbits, the $L O D$ is severely drifting in an unpredictable way. This drift is not identical from a series to another one. To determine these spurious drifts, we make use of the $L O D_{V L B I}$, obtained by time derivation of the UT1 VLBI values and given by the former C04 solution. Drift above 20 days in
$L O D_{G P S}-L O D_{V L B I}$ are then computed by low pass Vondrak filtering (99\% of the signal is let at 19 days) and removed from each GPS series.

Step 5: Sorting by increasing dates. For each EOP, the whole set of values are chronologically sorted.

Step 6: Running average. Data are averaged over successive 0.5 day time intervals. Using Lagrange interpolation we propagate the observed values to the averaged date. The average is weighted by the formal errors of the observed values. The averaged error or weight is also computed.

Step 7: Weighting change. We compute the offsets between the averaged series and the observed values (of Step 5), and the WRMS of the obtained time series. If for a given mean date, the offset is 2 times more than the RMS, then its weight is divided by 10 , and the averaging process of Step 6 is redone. From this gaussian weight we derive formal errors associated to the EOP estimates.

Step 8: High frequency filtering. Vondrak smoothing (Vondrak 1969, 1977) is applied in order to remove high frequency variations. Characteristics of the smoothing, ac- 


\begin{tabular}{llcccc} 
Time interval & & $\mathrm{x}, \mathrm{y}$ & $\mathrm{UT1}$ & LOD & DPSI, DEPS \\
\hline 1984-1993 & Smoothing coefficient & $10^{2}$ & $10^{0.7}$ & & $10^{0.5}$ \\
& 1\% remaining amplitude & $2.9 \mathrm{~d}$ & $4.8 \mathrm{~d}$ & & $5.2 \mathrm{~d}$ \\
& $99 \%$ remaining amplitude & $6.2 \mathrm{~d}$ & $10.3 \mathrm{~d}$ & & $11.2 \mathrm{~d}$ \\
\hline 1993-2006 & Smoothing coefficient & $10^{5}$ & $10^{5}$ & $10^{3}$ & $10^{0.5}$ \\
& 1\% remaining amplitude & $0.92 \mathrm{~d}$ & $0.92 \mathrm{~d}$ & $2 \mathrm{~d}$ & $5.2 \mathrm{~d}$ \\
& $99 \%$ remaining amplitude & $2 \mathrm{~d}$ & $2 \mathrm{~d}$ & $4.3 \mathrm{~d}$ & $11.2 \mathrm{~d}$ \\
\hline
\end{tabular}

Table 2: Smooting coefficient of the EOP

cording to the epoch of the solution, are reported in Table 2 .

Step 9: Interpolation (Lagrange). The filtered series are interpolated at 1 day intervals.

Step 10: Adding back the intermediate series. The final values are obtained by adding to the filtered and interpolated values i) the "intermediate" reference series, which had been removed in the Step 3 ii) the removed models during this step ("zonal tides" on UT1-TAI/LOD, precession-nutation offsets). The values UT1-TAI are then translated into the discontinuous series UT1UTC.

Step 11: Prediction and recording storage in the database. The next solution needs reference values covering the dates of the new observations. Therefore we extend the present series by a prediction, which is not described in this report. The solution associated with its prediction over 180 days is archived.

\section{Consistency with ITRF and ICRF}

A fundamental issue was solved : due to the separate determination of both celestial and terrestrial reference frames and EOP, there had been a slow degradation of the overall consistency. This has caused discrepancies at the level of 300 micro-arc-seconds between the current IERS C04 and the current ITRF realization. This was largely solved in the new solution by re-setting the $\mathrm{C} 04$ on the system linked to the newly issued ITRF2005 (Altamimi, 2006). Different hypotheses are adopted (see table 1) :

- UT1 and the celestial pole offsets (dPsi, dEps) provided by the IVS are consistent with the ICRF from 1984 to 2007.

- the polar motion components associated with the ITRF 2005 solution gives the direction of the CIP in the ITRF without any linear trend.

The differences between $05 \mathrm{C} 04$ solution and the former solutions are displayed on the Fig. 1.

\section{Other improvements}

- Model for nutation and UT1/LOD tidal variations have been updated according to the last IERS conventions (2003) : MHB 2000 for precession-nutation, Defraigne and Smits model for tidal variation in UT1/LOD (Step 3).

- Dimensions of tables have been significantly increased and double precision generalized to all parameters. This allows solution to be performed over 30 years in one run. 

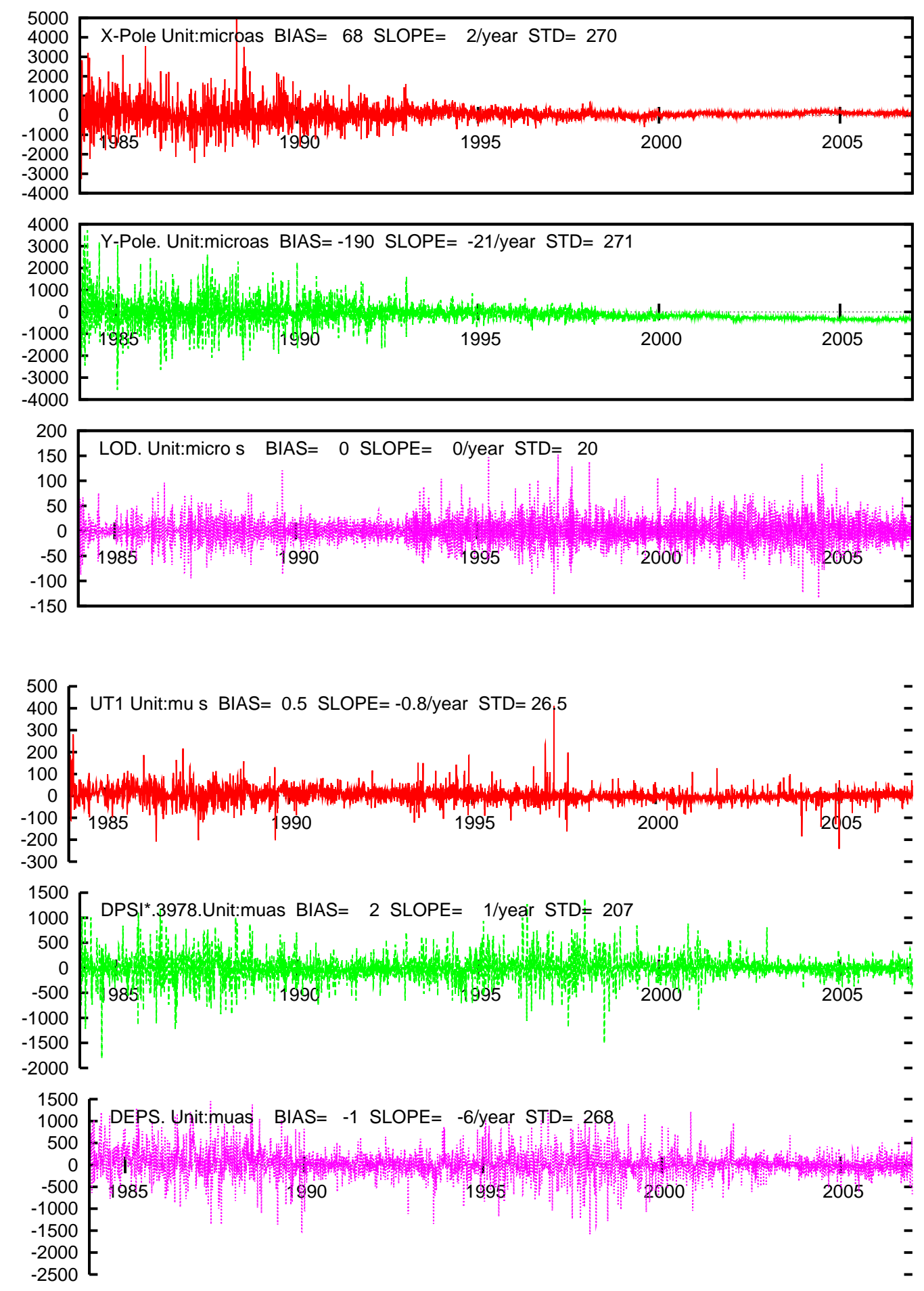

Figure 1: Differences 05 C04 - C04 from 1984 to 2007 for the Earth rotation parameters $x, y$, $L O D, U T 1, d p s i$, deps. Origin for the bias is 2000 . 
- A new approach for combination of LOD (GPS/SLR data) was developed, compatible with UT1-UTC (Step 4).

- Formal errors on EOP are estimated (Steps 6/7).

- The solution is automated.

Performances are significantly improved. This is illustrated by better RMS agreements of the differences between individual and the combined solution. We gain about $10 \mu$ as for long-term polar motion 3-4 $\mu$ s for UT1, and $40-50 \mu$ as for nutation offsets.

The possibility to make long-term computation over 20 years leads to an improved consistency and long-term stability of the solution.

\section{Agreement with GPS se- ries}

In the tables $3 / 4$ we compare GPS operational solution with respect to our combined solution and the IGS Rapid combined solution over the period [2000-2007]. Large bias disappear in the new pole solution, in particular for y-pole. Both solutions present comparable standard deviation (STD) with respect to GPS operational series.

\section{Agreement with IVS series}

A new combined IVS series, realesed in 2007, based on SINEX files concatenation is now available. This series shows a significant improvement with respect to the previous one.

New C04 series is now aligned on IVS series, supposed to be consistent with ICRF. We performed comparative analysis IVS / C04 with VLBI solution for UT1/Dpsi/Deps. Bias, slope and standard deviation with respect IVS operational series were computed over [1993-2007] and are reported in table 5 (C04) / 6 (IVS combined). The 05 C04 solution and new combined IVS series present in average comparable agreement with VLBI series : $6 \mu$ s for UT1, $70 \mu \mathrm{s}$ for $\left(d \psi \sin \varepsilon_{0}, d \varepsilon\right)$.

\section{Operational C04 solution}

The C04 series is fixed from 1/1/1962 to $1 / 1 / 2005$. It is recomputed from $1 / 1 / 2005$ every Tuesday and Thursday taking into account the last EOP determinations. This last part, undergoing changes from a computation to another, is called "operational C04 solution".

In table 7 we check the series entering our present combination. Note that except for SLR, the combination is not restricted to intra-technique combined series. We are not using IVS solution for 2 reasons :

- IVS combined latest value is several days late

- IVS combined does not take intensive sessions into account

- Our combination for the parameters dpsi,deps and UT1 seems to present closer agreement with IVS operational series than IVS combined series, according to the RMS value previously shown.

We do not combine exclusively IGS-Final or IGS-Rapid solutions because the last IGS$R$ values is available with a one day delay. CODE and GFZ provide the pole coordinates and LOD in a quasi real time.

\section{Summary}

The C04 solution has been improved : on one hand it is consistent with ICRF and ITRF 2005, on the other hand its parameters present a better accuracy. Pole motion and LOD is are as good as those of the official IGS combined series (30 $\mu$ as for pole motion, $15 \mu \mathrm{s}$ for LOD). The parameters $U T 1 / d \psi * \sin \varepsilon_{0}$ and $d \varepsilon$ are surprisingly 


\begin{tabular}{|c|c|c|c|c|c|c|c|c|c|c|c|}
\hline & CODE & JPL & GFZ & ESOC & NOAA & SIO & EMR & IAA & IGS-R & IGS-F & mean \\
\hline BIAS & 33 & 4 & -21 & 84 & -57 & 2 & 23 & -9 & 47 & -29 & 7.7 \\
\hline \pm & 3.4 & 2.5 & 2.4 & 5.1 & 8.9 & 3.3 & 3.7 & 9.0 & 3.2 & 1.6 & \\
\hline SLOPE & -1 & 11 & 12 & -8 & 21 & 7 & 5 & -7 & 5 & -7 & 3.8 \\
\hline \pm & 0.8 & 0.6 & 0.6 & 1.3 & 2.2 & 0.8 & 0.8 & 2.2 & 0.8 & 0.4 & \\
\hline WRMS & 67 & 51 & 48 & 105 & 171 & 69 & 66 & 176 & 61 & 32 & 84.6 \\
\hline
\end{tabular}

$\mathrm{x}$ pole : weighted rms (WRMS) and bias in $\mu a s$, slope in $\mu a s /$ year

\begin{tabular}{|c|c|c|c|c|c|c|c|c|c|c|c|}
\hline & CODE & JPL & GFZ & ESOC & NOAA & SIO & EMR & IAA & IGS-R & IGS-F & mean \\
\hline BIAS & -69 & 14 & -12 & 89 & 174 & 13 & -24 & -137 & -42 & -41 & -3.5 \\
\hline \pm & 2.8 & 2.5 & 3.2 & 4.3 & 191.9 & 3.3 & 4.2 & 10.2 & 3.2 & 1.5 & \\
\hline SLOPE & 9 & -29 & -21 & -29 & -90 & -11 & -11 & 58 & -11 & 58 & -7.7 \\
\hline \pm & 0.7 & 0.6 & 0.8 & 1.1 & 48.4 & 0.8 & 0.9 & 2.5 & 0.8 & 0.4 & \\
\hline WRMS & 57 & 51 & 65 & 90 & 3753 & 68 & 75 & 199 & 61 & 30 & 444.9 \\
\hline
\end{tabular}

y pole : weighted rms (WRMS) and bias in $\mu a s$, slope in $\mu a s /$ year

\begin{tabular}{|c|c|c|c|c|c|c|c|c|c|c|c|}
\hline & CODE & JPL & GFZ & ESOC & NOAA & SIO & EMR & IAA & IGS-R & IGS-F & mean \\
\hline BIAS & 23 & 5 & 3 & -18 & -1 & 19 & 21 & -1 & -3 & -3 & 4.5 \\
\hline \pm & 0.4 & 1.1 & 0.6 & 0.6 & 2.9 & 0.8 & 1.3 & 1.5 & 0.5 & 0.4 & \\
\hline SLOPE & -3 & -2 & -3 & -3 & -2 & -9 & -9 & -8 & -9 & -8 & -5.6 \\
\hline \pm & 0.1 & 0.3 & 0.2 & 0.2 & 0.8 & 0.2 & 0.3 & 0.4 & 0.2 & 0.1 & \\
\hline WRMS & 17 & 35 & 23 & 22 & 82 & 26 & 38 & 37 & 18 & 15 & 31.3 \\
\hline
\end{tabular}

LOD : weighted rms (WRMS) and bias in $\mu s$, slope in $\mu s /$ year

Table 3: IGS operational series - 05 C04 over [2000-2007]

in better agreement with VLBI series than the previous IVS official combined VLBI solution, especially for UT1. Their present accuracy is about $6 \mu \mathrm{s}$ for $U T 1$ and $70 \mu$ as for nutation offsets.

Acknowledgments We would like to express our gratitude to Dr. Jim Ray (NOAA, USA), Dr. Z. Altamimi (IGN, France), Dr. Z. Malkin (Pulkovo Observatory, St-Petersburg), Dr. C. Ma (GSFC, USA), Dr. W. Wooden (USNO, USA), Dr. E. Pavlis (USA, UMBC) and Dr. A Nothnagel (Bonn University) for their comments, validation and analyses of the new $05 \mathrm{C} 04$ combined solution.

\section{References}

[1] Defraigne P., and Smits I., 1999, Length of day variations due to zonal tides for an inelastic earth in non-hydrostatic equilibrium, Geophys. J. Int., 139, pp. 563-572.

[2] Gambis D., 2004, Monitoring Earth orientation using space-geodetic techniques : state-of-the-art and prospective, Journal of Geodesy 78, Issue 4-5, p 295-303.

[3] IERS2003, IERS CONVENTIONS (2003), IERS Technical note No. 32, Dennis D. McCarthy and Gérard Petit Eds. 


\begin{tabular}{|c|c|c|c|c|c|c|c|c|c|c|c|}
\hline & CODE & JPL & GFZ & ESOC & NOAA & SIO & EMR & IAA & IGS-R & IGS-F & mean \\
\hline BIAS & 58 & 36 & 8 & 110 & -22 & 43 & 61 & 40 & 75 & 0 & 40.9 \\
\hline \pm & 2.9 & 2.2 & 2.1 & 5.0 & 8.2 & 4.6 & 3.6 & 8.8 & 2.9 & 0.0 & \\
\hline SLOPE & -6 & 4 & 5 & -15 & 14 & -4 & -4 & -20 & -4 & -20 & -5.0 \\
\hline \pm & 0.6 & 0.5 & 0.5 & 1.1 & 1.8 & 0.9 & 0.7 & 2.0 & 0.6 & 0.0 & \\
\hline WRMS & 59 & 45 & 45 & 104 & 155 & 87 & 58 & 176 & 55 & 0 & 78.4 \\
\hline
\end{tabular}

$\mathrm{x}$ pole : weighted rms (WRMS) and bias in $\mu a s$, slope in $\mu a s /$ year

\begin{tabular}{|c|c|c|c|c|c|c|c|c|c|c|c|}
\hline & CODE & JPL & GFZ & ESOC & NOAA & SIO & EMR & IAA & IGS-R & IGS-F & mean \\
\hline BIAS & -22 & 51 & 16 & 110 & 590 & 39 & 19 & -53 & 6 & 0 & 75.6 \\
\hline \pm & 2.6 & 2.7 & 2.6 & 4.1 & 395.2 & 4.5 & 4.3 & 10.4 & 2.8 & 0.0 & \\
\hline SLOPE & 10 & -27 & -14 & -18 & -227 & -6 & -10 & 47 & -10 & 47 & -20.8 \\
\hline \pm & 0.6 & 0.6 & 0.6 & 0.9 & 85.8 & 0.9 & 0.8 & 2.3 & 0.6 & 0.0 & \\
\hline WRMS & 53 & 54 & 56 & 87 & 7763 & 87 & 69 & 205 & 54 & 0 & 842.8 \\
\hline
\end{tabular}

y pole : weighted rms (WRMS) and bias in $\mu a s$, slope in $\mu a s /$ year

\begin{tabular}{|c|c|c|c|c|c|c|c|c|c|c|c|}
\hline & CODE & JPL & GFZ & ESOC & NOAA & SIO & EMR & IAA & IGS-R & IGS-F & mean \\
\hline BIAS & 21 & -2 & -1 & -28 & -7 & 10 & -22 & -4 & -2 & 0 & -3.5 \\
\hline \pm & 0.7 & 1.6 & 0.7 & 1.4 & 4.2 & 1.1 & 1.2 & 1.7 & 0.6 & 0.0 & \\
\hline SLOPE & -3 & 0 & -2 & -1 & 0 & -7 & 0 & -8 & 0 & -8 & -2.9 \\
\hline \pm & 0.1 & 0.4 & 0.2 & 0.4 & 0.9 & 0.3 & 0.3 & 0.4 & 0.1 & 0.0 & \\
\hline WRMS & 18 & 36 & 15 & 34 & 65 & 23 & 23 & 34 & 12 & 0 & 26.0 \\
\hline
\end{tabular}

LOD : weighted rms (WRMS) and bias in $\mu s$, slope in $\mu s /$ year

Table 4: IGS operational series - IGS final over [ 2000-2007 ]

[4] Vondrak J., 1969, A contribution to the problem of smoothing observational data, Bull. Astron. Inst. Czech 20, p. 349.

[5] Vondrak J., 1977, Problem of smoothing observational data II, Bull. Astron. Inst. Czech 28, pp. 84-89. 


\begin{tabular}{|c|c|c|c|c|c|c|c|c|c|}
\hline & AUS & BKG & GSFC & IAA & SPBU & USNO & MAO & IVS & mean \\
\hline BIAS & 0.5 & 1.4 & 1.6 & 0.2 & -4.2 & 1.2 & -7.8 & -0.3 & -0.9 \\
\hline \pm & 0.3 & 0.1 & 0.1 & 0.1 & 0.3 & 0.1 & 0.5 & 0.1 & \\
\hline SLOPE & -0.2 & -0.1 & -1.1 & 0.0 & 0.4 & -0.6 & 1.1 & 0.0 & -0.1 \\
\hline \pm & 0.1 & 0.0 & 0.0 & 0.0 & 0.1 & 0.0 & 0.1 & 0.0 & \\
\hline WRMS & 6.0 & 5.1 & 4.0 & 4.5 & 8.2 & 3.1 & 7.6 & 5.2 & 5.5 \\
\hline
\end{tabular}

UT1. Units : $\mu \mathrm{s}$, slope in $\mu \mathrm{s} /$ year

\begin{tabular}{|c|c|c|c|c|c|c|c|c|c|}
\hline & AUS & BKG & GSFC & IAA & SPBU & USNO & MAO & IVS & mean \\
\hline BIAS & 16 & -21 & -16 & 2 & 10 & 7 & -23 & 5 & -2.5 \\
\hline \pm & 4.3 & 1.5 & 1.1 & 1.7 & 3.4 & 1.0 & 6.0 & 1.1 & \\
\hline SLOPE & -6 & -1 & -2 & 3 & -2 & -2 & 2 & -1 & -1.1 \\
\hline \pm & 0.9 & 0.4 & 0.3 & 0.4 & 0.8 & 0.2 & 1.6 & 0.3 & \\
\hline WRMS & 106 & 60 & 44 & 67 & 86 & 41 & 133 & 44 & 72.6 \\
\hline \multicolumn{10}{|c|}{$d \psi\left(\varepsilon_{0}\right)$. Units : $\mu$ as, slope in $\mu$ as/year }
\end{tabular}

\begin{tabular}{|c|c|c|c|c|c|c|c|c|c|}
\hline & AUS & BKG & GSFC & IAA & SPBU & USNO & MAO & IVS & mean \\
\hline BIAS & 24 & 14 & -19 & 17 & 18 & 4 & 25 & -1 & 10.2 \\
\hline \pm & 3.7 & 1.7 & 1.5 & 1.6 & 3.1 & 1.5 & 5.9 & 1.4 & \\
\hline SLOPE & -4 & -1 & -1 & -4 & -2 & -1 & 1 & -1 & -1.6 \\
\hline \pm & 0.8 & 0.4 & 0.4 & 0.4 & 0.7 & 0.4 & 1.4 & 0.3 & \\
\hline WRMS & 90 & 69 & 60 & 59 & 78 & 57 & 110 & 54 & 72.1 \\
\hline
\end{tabular}

Table 5: IVS operational series - C04 combined series over [ 1993-2007]: standard deviation, bias and slope 


\begin{tabular}{|c|c|c|c|c|c|c|c|c|c|}
\hline & AUS & BKG & GSFC & IAA & SPBU & USNO & MAO & IVS & mean \\
\hline BIAS & 0.4 & 1.6 & 1.8 & 0.2 & -4.2 & 1.6 & -9.3 & 0.0 & -1.0 \\
\hline \pm & 0.3 & 0.2 & 0.1 & 0.3 & 0.3 & 0.2 & 0.9 & 0.0 & \\
\hline SLOPE & -0.2 & -0.2 & -1.1 & -0.1 & 0.4 & -0.6 & 1.6 & 0.0 & -0.0 \\
\hline \pm & 0.1 & 0.0 & 0.0 & 0.1 & 0.1 & 0.0 & 0.2 & 0.0 & \\
\hline WRMS & 6.1 & 6.5 & 3.0 & 10.9 & 8.4 & 6.0 & 12.1 & 0.0 & 6.6 \\
\hline
\end{tabular}

UT1. Units : $\mu \mathrm{s}$, slope in $\mu \mathrm{s} /$ year

\begin{tabular}{|c|c|c|c|c|c|c|c|c|c|}
\hline & AUS & BKG & GSFC & IAA & SPBU & USNO & MAO & IVS & mean \\
\hline BIAS & 10 & -26 & -22 & -3 & 3 & 2 & -29 & -1 & -8.2 \\
\hline \pm & 4.4 & 1.4 & 1.2 & 2.3 & 3.7 & 1.4 & 6.8 & 0.0 & \\
\hline SLOPE & -5 & 0 & -1 & 4 & -1 & -1 & 3 & 0 & -0.1 \\
\hline \pm & 1.0 & 0.3 & 0.3 & 0.5 & 0.9 & 0.3 & 1.7 & 0.0 & \\
\hline WRMS & 113 & 56 & 47 & 89 & 101 & 54 & 119 & 0 & 72.4 \\
\hline
\end{tabular}

\begin{tabular}{|c|c|c|c|c|c|c|c|c|c|}
\hline & AUS & BKG & GSFC & IAA & SPBU & USNO & MAO & IVS & mean \\
\hline BIAS & 25 & 14 & -17 & 15 & 19 & 7 & 26 & 0 & 11.1 \\
\hline \pm & 3.9 & 1.4 & 1.2 & 2.3 & 3.9 & 1.3 & 8.3 & 0.0 & \\
\hline SLOPE & -4 & 0 & -2 & -3 & -3 & -1 & 1 & 0 & -1.5 \\
\hline \pm & 0.9 & 0.3 & 0.3 & 0.5 & 0.9 & 0.3 & 1.9 & 0.0 & \\
\hline WRMS & 100 & 57 & 46 & 91 & 106 & 52 & 123 & 0 & 71.9 \\
\hline
\end{tabular}

Table 6: IVS operational series - IVS combined series over [ 1993-2007]: standard deviation, bias and slope

\begin{tabular}{ll}
\hline VLBI standard AUS & VLBI standard BKG \\
VLBI standard GSFC & VLBI standard IAA \\
VLBI standard MAO & VLBI standard SPBU \\
VLBI standard USNO & \\
\hline VLBI intensive BKG & VLBI intensive GSFC \\
VLBI Intensive IAA & VLBI Intensive SPBU \\
VLBI intensive USNO & \\
\hline GPS IGS-Rapid & \\
GPS IGS-Final & \\
\hline Combined Laser ASI & Combined IGN (until 2006.0)
\end{tabular}

Table 7: EOP series used for the operational C04 solution (2005-today) 